\title{
Isolation and molecular characterisation of Mycobacterium bovis from raw milk in Tunisia
}

\author{
* Ben Kahla ${ }^{1,2}$, Boschiroli $\mathrm{ML}^{3}$, Souissi $\mathrm{F}^{1}$, Cherif $\mathrm{N}^{1}$, Benzarti $\mathrm{M}^{4}$, Boukadida $\mathrm{J}^{2}$, Hammami $\mathrm{S}^{1}$ \\ 1. Veterinary Research Institute of Tunisia. 20, Avenue Djebel Lakhdhar, La Rabta 1006 Tunis, Tunisia \\ 2. Microbiology and Immunology Laboratory, CHU Farhat Hached, Avenue IBN EL Jazzar, 4001 Sousse, Tunisia \\ 3. National and OIE/FAO Bovine Tuberculosis Reference Laboratories, Bacterial Zoonoses Unit - Animal Health \\ Laboratory- French Agency for Food, Environmental and Occupational health safety, 23 Avenue du Général-de-Gaulle, \\ 94706 Maisons-Alfort Cedex, France \\ 4. National Veterinary Medicine School, Sidi Thabet, Tunisia.
}

\begin{abstract}
Background: Consumption of raw milk and unpasteurized dairy products is common in Tunisia where bovine tuberculosis remains enzootic. We herein investigated the frequency of $M$. bovis isolation from raw milk.

Methods: Three hundred and six milk samples collected from 102 infected cows in different Tunisian regions were analysed. M. bovis isolates were further characterized by spoligotyping and variable number tandem repeat typing.

Results: A total of five (4.9\%) M. bovis strains exhibiting three different genotypes were isolated.

Conclusion: This study demonstrates that consumers of raw milk or derivatives in Tunisia are at high risk of zoonotic infection with $M$. bovis.

Keywords: Mycobacterium bovis; Tuberculosis; Raw milk, Zoonosis

African Health Sciences 2011; (S1): S2 - S5
\end{abstract}

\section{Introduction}

Bovine tuberculosis (BTB) caused by Mycobacterium bovis is a widespread zoonosis of a potential health hazard. Unpasteurized milk and dairy products prepared domestically by fermentation constitute the main source of human $M$. bovis exposure ${ }^{1}$. These types of products are still consumed in Tunisia where BTB remains enzootic. A national program for BTB control hs been implemented in Tunisia since $1984^{2}$. Nevertheless, the disease continues being prevalent mainly in the private sector which owns more than $70 \%$ of the cattle livestock ${ }^{3}$. Movement of infected cattle may be one of the major constraints in the control strategy in this sector. The aim of the present study was to show the frequency of $M$. bovis isolation from raw milk of infected cows in Tunisia in order to estimate the risk of food-borne transmission of M. bovis.

\author{
*Corresponding author \\ Imen Ben Kahla \\ Microbiology and Immunology Laboratory \\ CHU Farhat Hached \\ Avenue IBN EL Jazzar \\ 4001 Sousse, Tunisia \\ Tel: 0021697135718 \\ E-mail : imen.benkahla@hotmail.com
}

\section{Methods \\ Sampling}

Milk sampling was performed in 9 private farms recognized as BTB infected localized in three Tunisian governorates including Tunis (North region), Sousse and Monastir (Central regions), from October 2005 to January 2006. Farms were categorised according to the herd size: A ( $<5$ cattle), B (from 5 to 20 cattle) and $\mathrm{C}(>20$ cattle). Samples $(\mathrm{N}=306)$ were collected during milking from 102 Pie-Noire and Pie-Noire Holstein cows that were positive for the single cervical intradermal tuberculin test (SCITT), whether showing or not the clinical signs of tuberculosis and mastitis. We collected three specimens/animal with an interval of 15 days between each sampling. One $M$. bovis strain isolated from a human sample at the Microbiology and Immunology Laboratory, Farhat Hached Hospital, Sousse, Tunisia was included as positive control.

\section{Bacteriological and molecular analysis}

All samples were processed for $M$. bovis isolation in the veterinary research institute of Tunisia as previously described ${ }^{4}$. Briefly, a volume of $10 \mathrm{ml}$ of raw milk was decontaminated with $4 \%$ sodium hydroxide and then neutralized using concentrated hydrochloric acid. Suspensions were then centrifuged at $3000 \mathrm{~g}$ for $30 \mathrm{~min}$ and the sediment was 
inoculated onto Lowenstein-Jensen and Coletsos media and incubated at $37^{\circ} \mathrm{C}$ for 8 weeks. $M$. bovis characterization was performed using the ZiehlNeelsen staining, culture characteristics (growth rate, colony morphology), IS6110 and RD4 based-PCR, spoligotyping and variable number tandem repeat (VNTR) typing $5,6,9,10$. Spoligotype patterns were named according to the international spoligotype database nomenclature (http://www.mbovis.org).

\section{Ethics}

The experiments comply with the current laws of the country in which they were performed.

\section{Results}

Of the 102 SCIT'T positive cows included in our study, $5(4.9 \%)$ were detected as shedders $M$. bovis in the milk. These cows belong to the three farm categories i.e. category A (1 cow), B (1 cow) and C (3 cows) (table 1). In addition, 6 nontuberculosis mycobacteria were isolated and only one strain was identified as $M$. flavescens using the commercial kits Genotype Mycobacteria CM and AS (data not shown). The presence or absence of mycobacteria could not be confirmed for 28 of the raw milk cultures because of overgrowth by other contaminant microorganisms.

Table1: Geographical origin, herd size and sampling size from the nine farms included in this study

\begin{tabular}{llllll}
\hline Origin & Governorate & Category $^{\text {a }}$ & No. of cows & No. of milk No. of M. bovis \\
samples & isolates \\
\hline Farm 1 & Monastir & B & 2 & 6 & 0 \\
Farm 2 & Monastir & A & 2 & 6 & $1\left(\mathrm{Mb}^{\mathrm{b}} 1\right)$ \\
Farm 3 & Monastir & $\mathrm{B}$ & 4 & 12 & $1(\mathrm{Mb} 2)$ \\
Farm 4 & Monastir & $\mathrm{A}$ & 2 & 6 & 0 \\
Farm 5 & Monastir & $\mathrm{A}$ & 2 & 6 & 0 \\
Farm 6 & Monastir & $\mathrm{C}$ & 7 & 21 & 0 \\
Farm 7 & Sousse & $\mathrm{C}$ & 6 & 18 & 0 \\
Farm 8 & Tunis & $\mathrm{C}$ & 55 & 165 & $2(\mathrm{Mb3}, \mathrm{Mb} 4)$ \\
Farm 9 & Tunis & $\mathrm{C}$ & 22 & 66 & $1(\mathrm{Mb} 5)$ \\
Positive & Sousse & - & - & - & $1(\mathrm{Mb} 6)$ \\
control & & & & & \\
\hline
\end{tabular}

${ }^{\mathrm{a}}$ Farms were categorised according to the herd size: A ( $<5$ cattle), B (from 5 to 20 cattle) and C ( $>20$ cattle)

${ }^{\mathrm{b}} \mathrm{Mb}=$ M. bovis

To investigate the genetic diversity and relatedness of the $6 \mathrm{M}$. bovis isolates (5 cattle and 1 human isolates), genotyping using spoligotyping and VNTR typing techniques was carried out. A total of 3 different spoligopatterns were obtained (table 2). One spoligotype pattern (strain Mb1) had not been previously reported on the $M$. bovis database, and it was therefore designated SB1200. In addition to the deletion of spacers 3, 9, 16 and 39 to 43, characteristic features of cattle isolates ${ }^{9}$, this type was

characterized by the lack of spacers 28 and 29. The VNTR analysis further distinguished 4 different patterns. The human M. bovis strain (Mb6) included in this study as positive control showed the same spoligopattern (SB1093) and VNTR profile (5-4-24-3-2-10-3-11-6) as strain $\mathrm{Mb} 2$ isolated from raw milk. This strain was isolated from a cutaneous lesion of farmer women strongly suggesting an occupational exposure to $M$. bovis.

Table 2: Spoligotypes and variable number tandem repeat (VNTR) profile of the M.bovis isolates characterized in this study

\begin{tabular}{lccc}
\hline $\begin{array}{l}\text { M. bovis } \\
\text { isolate }\end{array}$ & $\begin{array}{c}\text { Spoligo- } \\
\text { pattern } \\
\text { designation }^{\mathrm{a}}\end{array}$ & $\begin{array}{c}\text { Spoligopattern } \\
\text { Spacer 1 43 }\end{array}$ & VNTR profile $^{\mathrm{b}}$ \\
\hline $\mathrm{Mb} 1$ & $\mathrm{SB} 1200$ & 11011111011111101111111111110011111111100000 & $5-8-4-4-3-2-12-3-7-6$ \\
$\mathrm{Mb} 2$ & $\mathrm{SB} 1093$ & 1100111101111110111101111111111111111100000 & $5-4-2-4-3-2-10-3-11-6$ \\
$\mathrm{Mb3}$ & $\mathrm{SB} 1093$ & 1100111101111110111101111111111111111100000 & $5-4-2-4-3-2-12-3-13-6$ \\
\hline
\end{tabular}




\begin{tabular}{lccc}
\hline $\begin{array}{l}\text { M. bovis } \\
\text { isolate }\end{array}$ & $\begin{array}{c}\text { Spoligo- } \\
\text { pattern } \\
\text { designation }^{\mathrm{a}}\end{array}$ & $\begin{array}{c}\text { Spoligopattern } \\
\text { Spacer 1 }\end{array}$ & VNTR profile $^{\mathrm{b}}$ \\
\hline $\mathrm{Mb} 4$ & $\mathrm{SB} 1003$ & 1101110101111110111111111110111111111100000 & $5-4-4-4-3-2-12-4-15-5$ \\
$\mathrm{Mb5}$ & $\mathrm{SB} 1003$ & 1101110101111110111111111110111111111100000 & $5-4-4-4-3-2-12-4-15-5$ \\
$\mathrm{Mb} 6$ & $\mathrm{SB1093}$ & 1100111101111110111101111111111111111100000 & $5-4-2-4-3-2-10-3-11-6$ \\
\hline
\end{tabular}

${ }^{a}$ www.mbovis.org

b $1=$ spacer present, $0=$ spacer absent

c The VNTR profile is defined by the number of tandem repeats in ETR-A, -B, -C, -D, -E,-F, QUB-11a, 11b, -3336 and -26 loci consecutively.

\section{Discussion}

Unfortunately, during the study period we did not have the opportunity to follow up the cows excreting M. bovis in the milk at post-mortem to further check udders and mammary lymph-nodes. This would be interesting for examining the correlation between $M$. bovis excretion in milk and the presence or absence tuberculous lesions. The fact that $M$. bovis was isolated from both small (category A) and large (category C) farms is of considerable importance particularly for category A farms. Indeed, the small quantity of produced milk may not be sold to dairy industry for pasteurization, but sold at retail and may be consumed raw or used for producing fermented dairy products. The exact relative contribution of $M$. bovis in the Tunisian human population is unknown. Nevertheless, twelve human extrapulmonary tuberculosis cases due to $M$. bovis including one multidrug resistant strain have previously been reported (Slim-Saidi L. unpublished data). The consumption of raw milk or derivatives was mentioned in six (60 $\%$ ) out of ten cervical lymphadenopathy cases. Our data support the premise that raw milk is a potential source of $M$. bovis transmission in Tunisia and thus particular vigilance is necessary. The frequency of $M$. bovis isolation reported here is higher than that reported in other developing countries ${ }^{7,8}$. The reason for this result may be due in part to the sample origin. Indeed, we collected milk samples only from positive SCITT cows while Srivastava el $\mathrm{al}^{8}$ included tuberculin negative and apparently healthy animals and Leite et $\mathrm{al}^{7}$ collected milk samples from retail markets.

Spoligotyping and VNTR analysis revealed the heterogeneity of the six $M$. bovis isolates (five cattle and one human isolate). Indeed, three spoligopatterns and four VNTR profiles were obtained (table 2). So far, however, it is not possible to make any epidemiological link between the different strains since our collection is limited in size and geographical representation. In addition, no data showing the genetic diversity of $M$. bovis population in Tunisia are available. According to the spoligotype database, the molecular characteristics of $M$. bovis strains described here are comparable, more likely, to molecular types of $M$. bovis strains isolated in European countries rather than those in African countries including neighbouring Algeria ${ }^{11}$. This finding needs to be further elucidated by larger molecular investigations.

\section{Conclusion}

As long as BTB remains enzootic in Tunisia, consumers of raw milk and unpasteurized dairy products may be exposed to $M$. bovis infection. Therefore, control campaigns in cattle in addition to public health education on the risk associated with the consumption of raw milk and fresh dairy products should be accentuated. Molecular epidemiological studies would be very helpful to increase our knowledge of $M$. bovis dissemination in Tunisia.

\section{Acknowledgement}

This work was supported by the Ministry of Agriculture and Hydrolic Resources, Tunisia.

We are grateful to Dr Moncef Rouis, Dr Nouri Zaddem, Dr Habib Lahouar, Dr Jamila Laarif, Dr Manoubi Souli and Dr Abdelwaheb Besbes for their help for sample collection.

\section{References}

1. Thoen C, LoBue P, de Kantor I. The importance of Mycobacterium bovis as a zoonosis. Vet Microbiol 2006; 112:339-345.

2. Cosivi O, Grange JM, Daborn CJ, Raviglione MC, Fujikura T, Cousins D, Robinson R A, Huchzermeyer HFAK, de Kantor I, Meslin FX. 
Zoonotic tuberculosis due to Mycobacterium bovis in developing countries. Emerg Inf Dis 1998; 4:5969.

3. Bahri, S, Kallel, A and Gouia, A. Lutte contre la tuberculose bovine: programme et réalisation. El Baytari 1991; 3:1-3.

4. Mishra A, Singhal A, Chauhan DS, Katoch VM, Srivastava K, Thakral SS, Bharadwaj SS, Sreenivas V, Prasad HK. Direct Detection and Identification of Mycobacterium tuberculosis and Mycobacterium bovis in Bovine Samples by a Novel Nested PCR Assay: Correlation with Conventional Techniques. J Clin Microbiol 2005; 43:5670-5678.

5. Vitale F, Capra G, Maxia L, Reale S, Vesco G, Caracappa S. Detection of Mycobacterium tuberculosis complex in cattle by PCR using milk, lymph node aspirates, and nasal swabs. J Clin Microbiol 1998; 36:1050-1055.

6. Huard RC, de Oliveira LC, Ray Butler W, van Soolingen D, and Ho JL. PCR-based method to differentiate the subspecies of the Mycobacterium tuberculosis complex on the basis of genomic deletion. I Clin Microbiol 2003; 41:1637-1650.

7. Leite CQF, Anno IS, De Andrade Leite SR, Roxo E, Morlock GP, and Cooksey RC. Isolation and identification of mycobacteria from livestock specimens and milk obtained in
Brasil. Mem Inst Oswaldo Cruz Rio de Janeiro 2003; 98: 319-323.

8. Srivastava K, Chauhan DS, Gupta P, Singh HB, Sharma VD, Yadav VS, Sreekumaran Thakral SS, Dharamdheeran JS, Nigan P, Prasad HK, and Katoch VM. Isolation of Mycobacterium bovis and Mycobacterium tuberculosis from cattle of some farms in north India - Possible relevance in human health. Indian J Med Res 2008; 128: 26-31.

9. Aranaz A, Liebana E, Mateos A, Dominguez L, Vidal D, Domingo M, Gonzolez O, RodriguezFerri EF, Bunschoten AE, Van Emden JDA, Cousins D. Spacer oligonucleotide typing of Mycobacterium bovis strains from cattle and other animals: a tool for studying epidemiology of tuberculosis. J Clin Microbiol 1996; 34:2734-2740.

10. Roring S, Scott A, Brittain D, Walker I, Hewinson G, Neill S, Skuce R. Development of VariableNumber Tandem Repeat typing of Mycobacterium bovis: comparison of results with those obtained by using existing exact tandem repeats and spoligotyping. J Clin Microbiol 2002; 40:21262133.

11. Sahraoui N, Müller B, Guetarni D, Boulahbal F, Yala D, Ouzrout R, Berg S, Smith NH, Zinsstag J. Molecular characterization of Mycobacterium bovis strains isolated from cattle slaughtered at two abattoirs in Algeria. BMC Veterinary Research 2009; 5:4. 